\title{
Quantum Remnant as Dark Energy and Dark Matter
}

\author{
Sang Pyo Kim* \\ Department of Physics, Kunsan National University, Kunsan 573-701, Korea and \\ Asia Pacific Center for Theoretical Physics, Pohang 790-784, Korea \\ Seoktae Koh $\mathrm{K}^{\dagger}$ \\ Institute of Theoretical Physics, Chinese Academy of Sciences, \\ P.O. Box 2735, Beijing 100190, China
}

\begin{abstract}
We study the quantum remnant of a scalar field protected by the uncertainty principle. The quantum remnant that survived the later stage of evolution of the universe may provide dark energy and dark matter depending on the potential. Though the quantum remnant shares some useful property of complex scalar field (spintessence) dark energy model, quantum fluctuations are still unstable to the linear perturbations for $V \sim \phi^{q}$ with $q<1$ as in the spintessence model.
\end{abstract}

*Electronic address: sangkim@kunsan.ac.kr

$\dagger$ Electronic address: skoh@itp.ac.cn 
The current observational data predicts that the present universe has recently been dominated by dark energy and dark matter in the latest evolution [1, 2]. The origin and property of dark energy and dark matter is one of the most challenging problems of this century. There have been many models and proposals to explain the nature of dark energy [3] and dark matter [4].

One of such attempts is to introduce a complex scalar field, equivalent to two real scalar fields with the same coupling constants, whose conserved charge leads to a centrifugal force to the potential that may provide dark energy, dubbed spintessence [5, 6]. It is pointed out that the spintessence model of complex scalar may lead to formation of Q-balls, a drawback for dark energy [7].

In this paper, we show that a real scalar field in semiclassical gravity may share some of useful properties of spintessence without forming Q-balls. In semiclassical gravity approach a quantum real scalar field is equivalent to a complex scalar field with a constraint from the quantization rule [8, 9, 10]. The basic idea is as follows: the minimum uncertainty relation satisfied by the field $\phi$ and the momentum $\pi_{\phi}$ [in units of $c=\hbar=1$,

$$
\Delta \phi \Delta \pi_{\phi} \sim \frac{m_{P}^{3}}{2}
$$

approximately leads to an effective potential

$$
V_{\mathrm{eff}}(\chi) \sim \frac{m_{P}^{6}}{8 a^{6} \chi^{2}}+V(\chi),
$$

where $\chi \sim \Delta \phi$. Here, the quantization rule or uncertainty principle in semiclassical gravity plays the role of the conserved charge of the spintessence model, the difference being that the Planck constant (set to $\hbar=1$ ) is a fundamental constant in the former whereas the charge is an arbitrary parameter in the latter.

Thus, the minimum energy of the effective potential may be called the quantum remnant of a dynamical scalar field. Note that the physical argument for the quantum remnant is the uncertainty principle. Any quantum field, contrary to the classical counterpart, cannot settle down to the potential minimum because fluctuations originated from the uncertainty principle lead to a non-zero ground state energy. Likewise, the same principle works in semiclassical gravity for cosmology. The quantum real scalar field cannot rest at the minimum of the classical potential, but it fluctuates around the potential minimum.

We now study quantum effects of a single real scalar field in the later evolution of the 
universe. In a spatially flat FRW universe with metric

$$
d s^{2}=-d t^{2}+a^{2}(t) d \mathbf{x}^{2}
$$

the homogeneous real scalar field $\phi$ has the Hamiltonian

$$
\mathcal{H}(t)=\frac{\pi_{\phi}^{2}}{2 a^{3}}+a^{3} V(\phi),
$$

where $\pi_{\phi}=a^{3} \dot{\phi}$. The equation of motion for $\phi$ is given by

$$
\ddot{\phi}+3 H \dot{\phi}+V^{\prime}=0
$$

where $H=\dot{a} / a$ is the Hubble constant and a prime denotes derivative with respect to $\phi$. The homogeneous Friedmann equation is

$$
H^{2}=\frac{8 \pi}{3 a^{3} m_{P}} \mathcal{H}
$$

However, in semiclassical gravity, the classical equations are replaced by the Schrödinger equation for $\phi$

$$
i \frac{\partial}{\partial t} \Psi(\phi, t)=\mathcal{H}(t) \Psi(\phi, t)
$$

and by the semiclassical Friedmann equation

$$
H^{2}=\frac{8 \pi}{3 a^{3} m_{P}}\langle\mathcal{H}\rangle_{\Psi}
$$

For a convex potential $V$, where the curvature is positive, a generic Gaussian wave function may approximate the exact state near the potential minimum [8]:

$$
\Psi(\phi)=\frac{1}{\left(2 \pi \chi^{2}\right)^{1 / 4}} \exp \left[-\left(\frac{1}{4 \chi^{2}}-i \frac{\pi_{\chi}}{2 \chi}\right) \phi^{2}\right] .
$$

Here, $\pi_{\chi}=a^{3} \dot{\chi}$, and the auxiliary variables satisfy the dispersions $\chi=\Delta \phi$ and $\pi_{\chi}=\Delta \pi_{\phi}$, respectively. Now the Dirac action principle leads to the effective action

$$
\mathcal{H}_{\mathrm{eff}}=\frac{\pi_{\chi}^{2}}{2 a^{3}}+\frac{m_{P}^{6}}{8 a^{6} \chi^{2}}+a^{3}\langle V(\phi)\rangle
$$

The origin of the centrifugal potential, the second term, in Eq. (10) is either the quantization rule or the uncertainty principle. Hence, the semiclassical Friedmann equation reads

$$
H^{2}=\frac{8 \pi}{3 a^{3} m_{P}} \mathcal{H}_{\mathrm{eff}}
$$


and the field equation becomes

$$
\ddot{\chi}+3 H \dot{\chi}-\frac{m_{P}^{6}}{4 a^{6} \chi^{3}}+\frac{\partial\langle V\rangle}{\partial \chi}=0 .
$$

In the oscillator representation, the Gaussian wave function is the ground state of the annihilation and the creation operators that are dimensionless,

$$
\begin{aligned}
\hat{a} & =\frac{\varphi^{*}}{m_{P}^{3}} \hat{\pi}_{\phi}-\frac{1}{m_{P}^{3}} \pi_{\varphi}^{*} \hat{\phi} \\
\hat{a}^{\dagger} & =\frac{\varphi}{m_{P}^{3}} \hat{\pi}_{\phi}-\frac{1}{m_{P}^{3}} \pi_{\varphi} \hat{\phi}
\end{aligned}
$$

where $\pi_{\varphi}=a^{3} \dot{\varphi}$ and a complex auxiliary field $\varphi$ satisfies the equation of motion

$$
\ddot{\varphi}+3 H \dot{\varphi}+V^{\prime}=0 .
$$

The commutation relation $\left[\hat{a}, \hat{a}^{\dagger}\right]=1$, or the quantization rule yields the condition

$$
\pi_{\varphi}^{*} \varphi-\varphi^{*} \pi_{\varphi}=i m_{P}^{3}
$$

The auxiliary field $\chi$ is the amplitude of the complex scalar field, $\varphi=\chi e^{-i \theta}$ and the quantization rule (16) becomes

$$
Q=2 a^{3} \chi^{2} \dot{\theta}=m_{P}^{3}
$$

Note that Eq. (17) has the same form as the charge conservation in the spintessence model [5, 6]. The centrifugal potential from the quantization, physically speaking, the uncertainty principle, prevents the field $\chi$ from falling to the classical minimum of $V$. Instead, it eventually reaches the limit cycle [8]

$$
\frac{m_{P}^{6}}{4 a^{6} \chi^{3}}=\frac{\partial\langle V\rangle}{\partial \chi} .
$$

To investigate the later evolution of quantum remnants, we need to check the equation of state. The energy density and the pressure for the zero-mode of quantum scalar field are given by

$$
\begin{aligned}
& \rho=\frac{1}{a^{3}}\langle\mathcal{H}\rangle=\frac{1}{2} \dot{\chi}^{2}+\frac{m_{P}^{6}}{8 a^{6} \chi^{2}}+V(\chi), \\
& p=\frac{1}{2} \dot{\chi}^{2}+\frac{m_{P}^{6}}{8 a^{6} \chi^{2}}-V(\chi) .
\end{aligned}
$$




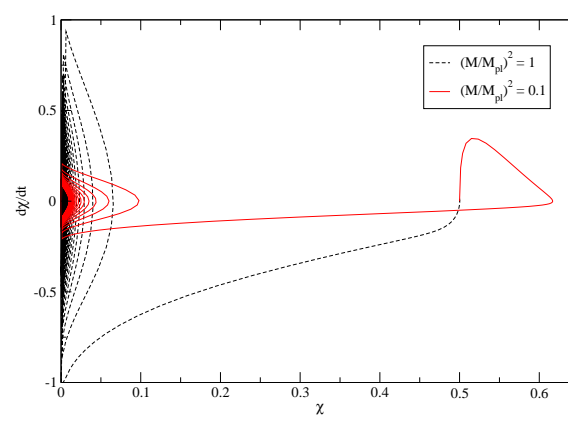

(a) Phase space

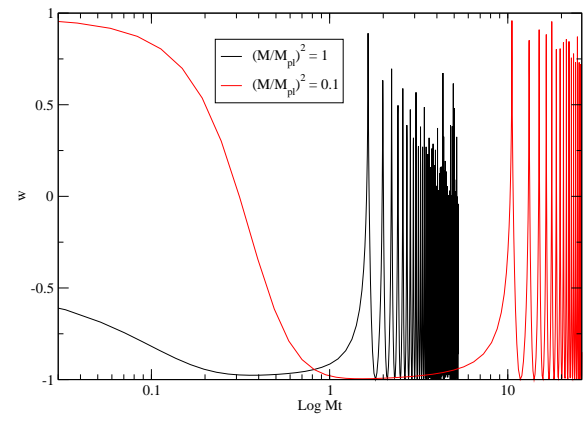

(b) Evolution of equation of state

parameter $w$

FIG. 1: (1a) depicts the evolution of fields in phase space for $V=M^{4-q} \chi^{q} / q$ where $q=1 / 2$ and $(1 \mathrm{~b})$ plots the evolution of equation of state parameter $w$

Then the equation of state around the limit cycle is

$$
w=\frac{p}{\rho} \simeq \frac{\chi V^{\prime}-2 V}{\chi V^{\prime}+2 V},
$$

where we have used Eq. (18). The $w$ should be less than $-1 / 3$ for dark energy. For a power-law potential, $V(\chi) \sim \chi^{q}$, this constraint restricts $q<1[6,6]$.

For a general concave potential with negative curvature, we give a simple argument based on the uncertainty principle. Roughly speaking, the field operator and the momentum operator satisfying the minimum uncertainty relation

$$
\chi \pi_{\chi} \sim \frac{m_{P}^{3}}{2}
$$

approximately leads to an effective potential

$$
V_{\mathrm{eff}}(\chi) \sim \frac{m_{P}^{6}}{8 a^{6} \chi^{2}}+V(\chi) .
$$

Therefore, the limit cycle is a consequence of the uncertainty principle.

In Figs. 1 and 2, we plot the evolution of $\chi$ in phase space and of $w$ for $V(\chi)=2 M^{7 / 2} \chi^{1 / 2}$ and $m^{2} \chi^{2} / 2$, respectively. It is numerically confirmed in Figs. 1a and $2 \mathrm{a}$ that a later time, there is a barrier due to the centrifugal potential term in Eq. (23) which prevents $\chi$ from falling to the potential minimum and that $\chi$ approaches to the limit cycle at $\chi \simeq \chi_{c}$, where $\chi_{c}$ is the value satisfying the condition (18). While $\langle w\rangle=0$ at limit cycle for $V(\chi) \sim \chi^{2}$ in Fig. 2b, $\langle w\rangle<-\frac{1}{3}$ for $V \sim \chi^{1 / 2}$ in Fig. 1b where we have performed time average over equation of state parameter at limit cycle. As discussed for a complex scalar field in Ref. 


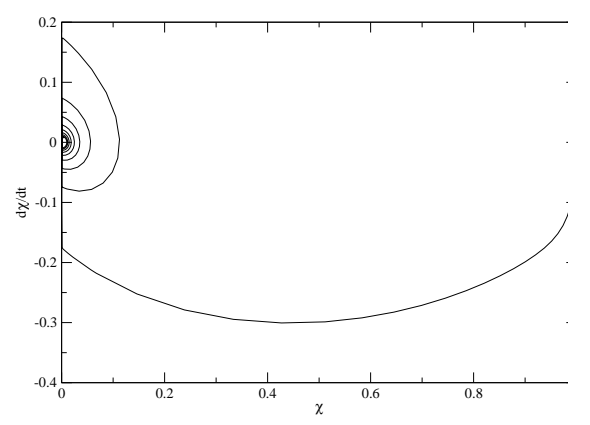

(a) Phase space

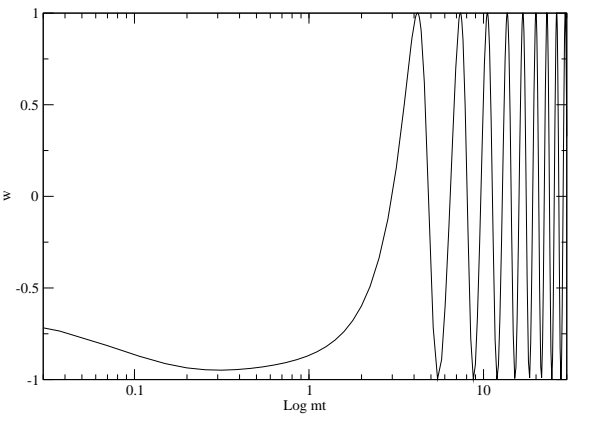

(b) Evolution of equation of state parameter $w$

FIG. 2: (2a) depicts the evolution of fields in phase space for $V=m^{2} \chi^{2} / 2$ with $m / m_{P}=1$ and (2b) plots the evolution of equation of state parameter $w$

[6], while the power-law potential with $q=2$ can play the role of dark matter, the power-law potential with $q<1$ can be considered as dark energy.

A few comments are in order. First, in discussing the Hamiltonian of the quantum scalar field, we assumed its ground state to be approximately described by the Gaussian wave function. It is believed to be true in general for a convex potential, especially $V \sim \phi^{q}$ with $q \geq 2$. However, for a (concave) power-law potential with $q<1$ it may not hold because of a singular behavior around $\phi=0$. In order to apply the approximate Gaussian wave function for the power law potential with $q<1$, we can consider the following form potential as in [11]

$$
V(\phi)=\frac{A}{q}\left[\left(\frac{\phi^{2}}{\phi_{c}^{2}}+1\right)^{(1 / 2) q}-1\right],
$$

where $A$ is a dimensionful parameter. At $\phi \gg \phi_{c}$, the potential $V(\phi)$ can be approximated by the power-law potential $V(\phi) \simeq \phi^{q}$, and if $\phi \ll \phi_{c}, V(\phi) \simeq \phi^{2}$. Since $V^{\prime}(\phi)$ is not singular any longer around $\phi=0$ even $q<1$, we may apply the Gaussian wave function approximately to describe the ground state and take $\phi_{c}$ as the value that satisfy the limit cycle condition (18) .

Next, we discuss the instability problem. The field $\phi(t, \mathbf{x})$ is decomposed into the homogeneous part, $\phi_{0}(t)$, which satisfies the equation of motion (5), and fluctuations $\delta \phi(t, \mathbf{x})$, which can be expressed in the Fourier transform as

$$
\delta \phi(t, \mathbf{x})=\int \frac{d^{3} k}{(2 \pi)^{3 / 2}}\left[\hat{a} \varphi_{k}(t) e^{-i \mathbf{k} \cdot \mathbf{x}}+\hat{a}^{\dagger} \varphi_{k}^{*}(t) e^{i \mathbf{k} \cdot \mathbf{x}}\right] .
$$


The expression $\hat{a}$ and $\hat{a}^{\dagger}$ can be obtained from Eqs. (13) and ( 14). Then $\varphi_{k}$ satisfy the equations of motion

$$
\ddot{\varphi}_{k}+3 H \dot{\varphi}_{k}+\left(\frac{k^{2}}{a^{2}}+V^{\prime \prime}\right) \varphi_{k}=0
$$

where we have neglected the metric perturbations. If $V^{\prime \prime}$ is assumed to include oscillating terms (for example, $V^{\prime \prime} \approx V_{0}^{\prime \prime}+V_{2}^{\prime \prime} \cos 2 \omega t$ and $\omega$ is an oscillating frequency [12]), the above equation reduces to the form of Mathieu equation [13, 14, 15]

$$
\frac{d^{2}}{d z^{2}} \varphi_{k}+\left(\mathcal{A}_{k}-2 \mathcal{B} \cos z\right) \varphi_{k}=0
$$

where $z=\omega t, \mathcal{A}_{k}=\frac{4}{\omega^{2}}\left(k^{2}+2 V_{0}^{\prime \prime}\right)$ and $\mathcal{B}=-\frac{2 V_{2}^{\prime \prime}}{\omega^{2}}$ and we have ignored cosmic expansion. For a symmetric power-law potential with $p \geq 2$, there exist stable solutions even in small scale regions [16]. For a power-law potential with $q<1$, however, numerical calculations show that oscillating scalar fields which have negative pressure are unstable to the growth of inhomogeneities on small scales [16] and on large scales [12]. This implies that instabilities are inevitable as far as the power-law potential is concerned with $q<1$ which provides negative pressure. In this sense such a potential may not be suitable for driving cosmic acceleration.

However, the Gaussian wave function (9) may be not appropriate to apply to the concave potential or potential (24) although we assume it would hold approximately in the present work. We have to solve the Schrödinger equation (77) for a concave potential or potential (24) and then improve the Gaussian wave function using a variational method. And also in (26), we can take into account higher order terms in $V^{\prime \prime}$

$$
\ddot{\varphi}_{k}+3 H \dot{\varphi}_{k}+\left(\frac{k^{2}}{a^{2}}+\sum_{n=0}^{\infty} \frac{1}{2^{n} n !}\left\langle\varphi_{k}^{2}\right\rangle V^{(2 n+2)}\right) \varphi_{k}=0
$$

where

$$
\left\langle\varphi_{k}^{2}\right\rangle=\frac{1}{2 \pi^{2}} \int d k k^{2}\left|\varphi_{k}\right|^{2}
$$

and $V^{(n)}=d^{n} V / d \phi^{n}$. It is necessary to find the role of higher order quantum corrections to the instability problem. We shall leave these things to the future work.

In summary, we have studied the dynamics of a quantum real scalar field that obeys the uncertainty principle and discussed the possibility of its quantum remnant for dark matter and dark energy at later evolution. It is shown that the uncertainty principle, by 
preventing the quantum scalar field from rolling to the potential minimum, can drive the cosmic acceleration in the recent era. In fact, the uncertainty principle plays the similar role of the Hubble friction in oscillating dark energy model or the centrifugal potential in complex scalar fields (spintessence) model. If the quantum remnants survived to the present era, those can play the role of dark matter $(w=0)$ or dark energy $(w<-1 / 3)$ depending on the shape of the potential. However, although no Q-balls are formed in this model, quantum fluctuations are unstable to the growth of the inhomogeneities for the power-law potential with $q<2$ which provides negative pressure. As far as instability is concerned, the model may not be suitable as a candidate for dark energy but is not still excluded as a viable candidate for dark matter. So it would be interesting to study whether the improved Gaussian wave function by the variational method and thereby higher order quantum corrections to the potential may resolve the instability problem or not.

\section{Acknowledgments}

S. K. would like to appreciate the hospitality of Kunsan National University, where this paper was completed, and Center for Quantum Space Time (CQUeST) at Sogang University. This work was supported by Korea Science Engineering Foundation (KOSEF) grant funded by the Korea government (MOST) (No. F01-2007-000-10188-0).

[1] S. Perlmutter et al. [Supernova Cosmology Project Collaboration], Astrophys. J. 517, 565 (1999) arXiv:astro-ph/9812133; A. G. Riess et al. [Supernova Search Team Collaboration], Astron. J. 116, 1009 (1998) arXiv:astro-ph/9805201.

[2] E. Komatsu et al. [WMAP Collaboration], arXiv:0803.0547 [astro-ph]; J. Dunkley et al. [WMAP Collaboration], arXiv:0803.0586 [astro-ph].

[3] P. J. E. Peebles and B. Ratra, Rev. Mod. Phys. 75, 559 (2003) arXiv:astro-ph/0207347; T. Padmanabhan, Phys. Rept. 380, 235 (2003) arXiv:hep-th/0212290]; E. J. Copeland, M. Sami and S. Tsujikawa, Int. J. Mod. Phys. D 15, 1753 (2006) arXiv:hep-th/0603057.

[4] G. Bertone, D. Hooper and J. Silk, Phys. Rept. 405, 279 (2005) arXiv:hep-ph/0404175.

[5] J.-A. Gu and W.-Y. Hwang, Phys. Lett. B 517, 1 (2001). 
[6] L. A. Boyle, R. R. Cadwell and M. Kamionkowski, Phys. Lett. 545, 17 (2002).

[7] S. Kasuya, Phys. Lett. B 515, 121 (2001).

[8] D. Bak, S. P. Kim, S. K. Kim, K.-S. Soh, and J. H. Yee, Phys. Rev. D 59, 027301 (1998).

[9] S. P. Kim, Phys. Rev. D 55, 7511 (1997).

[10] S. P. Kim, J.-Y. Ji, H.-S. Shin and K.-S. Soh, Phys. Rev. D 56, 3756 (1997).

[11] T. Damour and V. F. Mukhanov, Phys. Rev. Lett. 80, 3440 (1998) arXiv:gr-qc/9712061.

[12] M. C. Johnson and M. Kamionkowski, arXiv:0805.1748 [astro-ph].

[13] J. H. Traschen and R. H. Brandenberger, Phys. Rev. D 42, 2491 (1990).

[14] L. Kofman, A. D. Linde and A. A. Starobinsky, Phys. Rev. Lett. 73, 3195 (1994) arXiv:hep-th/9405187.

[15] L. Kofman, A. D. Linde and A. A. Starobinsky, Phys. Rev. D 56, 3258 (1997) arXiv:hep-ph/9704452.

[16] A. Taruya, Phys. Rev. D 59, 103505 (1999) arXiv:hep-ph/9812342.

[17] S. Tsujikawa, Phys. Rev. D 61, 083516 (2000) arXiv:hep-ph/0003252. 